\title{
Lie Symmetries, Exact Solutions and Hidden Symmetries of a Class of Kuramoto Sivashinsky(KS) Equations
}

\author{
J E Okeke, O C Collins
}

\begin{abstract}
We study a class of non-linear partial differential equations, which describes the longitudinal motion of an elasto-plastic bar and anti-plane shearing deformation. In order to systematically explore the mathematical structure and underlying physics of the elasto-plastic flow in a medium, we generate all the geometric vector fields of the model equations. Using the classical Lie group method, it is shown that this equation does not admit space dilation type symmetries for a specific parameter value. The symmetry reductions and exact solutions to this equation are derived.
\end{abstract}

Index Terms - Lie Symmetries, invariants, and hidden symmetries

\section{INTRODUCTION}

Partial differential equations (PDEs) (Ames, 1972) are widely used to describe complex phenomena in various fields of sciences, especially in physical sciences. Therefore, solving partial differential equation problems plays an important role in sciences. The Lie group method is one of the most powerful and fundamental methods used to determine invariant solutions of differential equations. It is applicable to both linear and nonlinear differential equations. The fundamental basis of this method is that when a differential equation is invariant under a Lie group of transformations (Olver, 1993), a reduction transformation exists. For PDEs with two independent variables, a single group reduction transforms the PDEs into ordinary differential equations (ODEs), which are generally easier to solve. Kuramoto-Sivashinsky equation introduced by Kuramoto in 1976 , is one of the simplest physically, interesting spatially extended nonlinear systems that displays spatiotemporally chaotic behaviour. The equation is used to model stability of laminar flame fronts, phase turbulence in chemical oscillations and phase dynamics in reaction discusion systems (Kuramoto et. al., 1976), (Sivashinsky, Nicolaenko \& Zaleski, 1977).

In this paper we are concerned with the following class of KS equations

$u_{t t}+\omega u_{x x x x}-\gamma\left(u_{x}^{\mathrm{n}}\right)_{x}=0$,

J E Okeke, Department of Mathematics, Chukwuemeka Odumegwu Ojukwu University, Anambra State, Nigeria

O C Collins, Department of Mathematics, University of Nigeria, Nsukka, Enugu State, Nigeria where $\alpha, \gamma$ are constants and $\mathrm{n}>0$. This is a modified nonlinear wave equation introduced by Z. J. Yang and G. W. Chen, (Yang \& Chen 2000). The equation (1) is associated with many equations. For example, in (Peire \& An, 1995) a nonlinear wave equation

$u_{t t}+\omega u_{x x x y}-\gamma\left(u_{x}^{2}\right)_{x}=0$,

where $\mathrm{u}(\mathrm{x}, \mathrm{t})$ is the longitudinal displacement, $\alpha>0, y \neq 0$ are real numbers was presented.

It is used to study some problems about vertical vibration of one dimensional elasticity pole and two dimensional anti-plane shear in the weak nonlinear analysis of micro-structure model in the elasticity and plasticity. Furthermore, the instability of its special solution and ordinary stain solution were studied in (Peire \& An, 1995). In (Chen \& Yang., 2000), (Zhang \& Chen, 2003)., the authors considered the generalized equation of equation (2) and proved the existence and uniqueness of the global generalized solution and the global classical solution of several initial boundary value problems by the contraction mapping principle. The sufficient conditions of the nonexistence of the solution were also given. Z. Y. Yan (Yan, 2000). studied the equation (1) with the viscous damping term, by using the direct reduction method and obtained four new explicit solutions in the case of $n=2$. The work of (Yan, 2000). was extended by (Wu, \& Fan, 2007) via the same method and presented the solutions for the equation for $n \geq 3$. Since the nonlinear wave equation (1) has extensive physical applications in elasticity and plasticity, it is meaningful to study and investigate more new solutions of the equation.

In this paper, we have studied the equation via Lie symmetry analysis to generate more new solutions of the equation. Furthermore, the hidden symmetry approach is applied to generate new solutions that would not be predicted from the Lie point symmetries of the original wave equations.

The paper is structured as follows. In Section II, we present some basic operators and definitions which shall be used in our work. In Section III, the Lie point symmetries of equation (1) are presented. In Section IV, symmetry reductions and exact solutions are discussed. In Section $\mathrm{V}$, the hidden symmetries are obtained. Discussions and concluding remarks are given in Section VI.

\section{PRELIMINARIES}

We present some basic notations, operators and definitions to be used in our work, which are taken from (bragimov, 1994)., 
(Kara \& Mahomed., 1997), (Naz, Mahomed \& Mason, 2008) (Okeke, Narain, \& Govinder, 2019).

\section{Definition 2.1.}

A $k^{\text {th }}$ - order $(\mathrm{k} \geq 1)$ System $E^{\sigma}$ of s partial diff erential equations of $n$ independent variables $x^{i}{ }_{v} i=1,2, \ldots \ldots n$ and $m$-dependent variables $u^{\infty}: a=1,2 \ldots \ldots m$ is defined by;

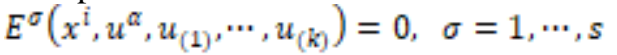

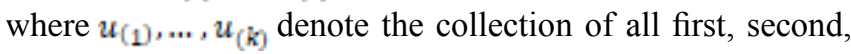
..., kth-order partial derivatives.

\section{Definition 2.2.}

The Euler-Lagrangian operator is defined by

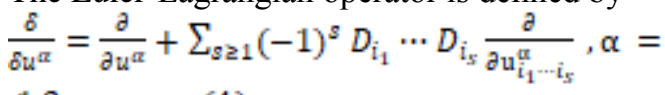
$1,2, \ldots, \mathrm{m}$

where

$D_{i}=\frac{\partial}{\partial x^{i}}+u_{i}^{\alpha} \frac{\partial}{\partial u^{x}}+u_{i f}^{E} \frac{\partial}{\partial u_{j}^{\alpha}}+\cdots, \quad i=$

$1,2, \ldots, \mathrm{n}$

is the total derivative operator with respect to $x^{i}$

Definition 2.3.

The Euler-Lagrangian equations, associated with (3) are the equations

$\frac{\partial L}{\partial u^{x}}=0, \alpha=1,2, \ldots, \mathrm{m}$

where $\mathrm{L}$ is referred to as a Lagrangian of (3).

Definition 2.4.

A Lie Backlund operator $\mathrm{X}$ is defined by

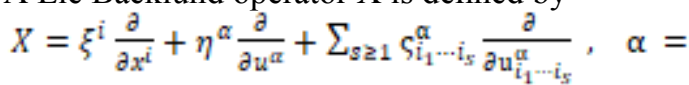

$1,2, \ldots x \mathrm{~m}$

(7)

where $S_{i_{1} \cdots \hat{i}_{s}}^{\mathbb{a}}$ are given as

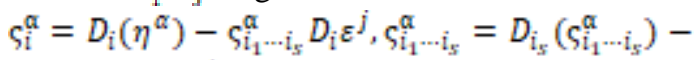
$\mathrm{u}_{j_{1}-i_{x-1}^{i}}^{\mathrm{d}} D_{\mathrm{i}_{x}}\left(\varepsilon^{j}\right), s \geq 1$.

The Lie point symmetry of equation (3) is a generator $\mathrm{X}$ of the form (7) that satisfies

$$
X^{[k]} F_{\mid F=0}=0,
$$

where $X^{[k]}$ is the $k t$ th prolongation of $\mathrm{X}$ i.e., $X^{[K]}=\xi^{i}(x, u) \frac{\partial}{\partial x^{i}}+\eta^{\alpha}(x, u) \frac{\partial}{\partial u^{x}}+\xi_{i}^{\alpha}\left(x, u, u_{[1}\right) \frac{\partial}{\partial u_{i}^{\alpha}}+$

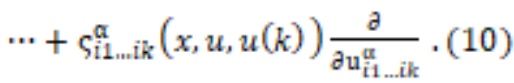

\section{LIE POINT SYMMETRIES}

In this section, we present the Lie point symmetry generators admitted by (1). The Lie point symmetries admitted by (1) are generated by a vector field of the form

$X=\xi^{1}(t, x, u) \frac{\partial}{\partial t}+\xi^{2}(t, x, u) \frac{\partial}{\partial x}+\eta(t, x, u) \frac{\partial}{\partial u^{\prime}}$

The operator X satisfies the Lie symmetry condition (Kara, \& Mahomed, 1997) ), (Okeke, Narain, \& Govinder, 2018) $\left.X^{[4]}\left[u_{t t}+\omega u_{x x x x}-\gamma\left(u_{x}^{n}\right)_{x}\right]\right]_{\mathbb{d} \mathbb{b}}=0$,

where $X^{[4]}$ is the fourth prolongation of the operator $\mathrm{X}$ and can be computed from (10).
Expansion and separation of (12) with respect to the powers of different derivatives of $\mathrm{u}$ yields an overdetermined system in the unknown coefficients $\xi^{1}{ }_{0} \xi^{2}$ and $\eta$. Solving the overdetermined system for $\xi^{1}(t, x, u), \xi^{2}(t, x, u)$ and $\eta(t, x, w)$ we obtain

$\xi^{1}(t, x, w)=C_{1}+t C_{\mathrm{a}}, \xi^{2}(t, x, u)=C_{2}+\frac{1}{2} x C_{\mathrm{g}}$,

$\eta(t, x, u)=\frac{1}{2}\left(\frac{n-3}{n-1}\right) u C_{9}+C_{4}+t C_{5}$ (13)

where $C_{1}, C_{2}, C_{\mathrm{g}}, C_{4}$ and $C_{5}$ are arbitrary constants. Solutions (13) prompt the consideration of special cases of equation (1) namely, (i) $n=1$ and (ii) $n=3$. Hence, we shall also

discuss the symmetries based on these two cases. Equations (13) lead to a five-dimensional

Lie algebra spanned by the following basis

$X_{11}=\frac{\partial}{\partial x}, X_{12}=\frac{\partial}{\partial t}, X_{1 a}=\frac{x}{2} \frac{\partial}{\partial x}+t \frac{\partial}{\partial t}+\frac{1}{2}\left(\frac{n-a}{n-1}\right) u \frac{\partial}{\partial u}$,

$X_{14}=\frac{\partial}{\partial u}, X_{15}=t \frac{\partial}{\partial u}$,

The commutation relation between these five operators is presented in Table 1, where each

entry, $A_{i j}$, constitutes the Lie Bracket $\left[X_{1 j}, X_{1 j}\right]$ of two infinitesimal generators from (14) for

$1 \leq i_{i} j \leq 5$

Special cases

(i) $\mathbf{n}=\mathbf{1}$

This case gives rise to the linear wave equation

$u_{t t}+c u_{x x x x}-\gamma u_{x x}=0$.

Equation (15) admits four Lie point symmetries given by

$X_{21}=\frac{\partial}{\partial x}, \quad X_{22}=\frac{\partial}{\partial t}, \quad X_{2 a}=u \frac{\partial}{\partial u}$.

as well as an infinite symmetry $X_{24}=F_{1}(t, x) \frac{\partial}{\partial u}$, where $F_{1}(t, x)$ is a solution of equation (15) and hence called the solution symmetry. This symmetry always arises in the event that the equation in question is linear. We observe the loss of invariance under dilations in space and time $\left(X_{13}\right)$. This loss is indeed well compensated by the infinite-dimensional Lie subalgebra $\left(X_{24}\right)$.

(ii) $\mathbf{n}=\mathbf{3}$

In this case we obtain a well known PDE, the modified Boussinesq equation given by (Ghil \& Paldor, 1994)

$u_{t t}+\omega u_{x x x x}-\gamma\left(u_{x}^{3}\right)_{x}=0$.

which was presented in the famous Fermi-Pasta-Ulam problem. The symmetries are

$X_{21}=\frac{\partial}{\partial t}, X_{22}=\frac{\partial}{\partial x}, X_{a 2}=\frac{x}{2} \frac{\partial}{\partial x}+t \frac{\partial}{\partial t}, X_{24}=\frac{\partial}{\partial u}, X_{25}=$ $t \frac{\partial}{\partial u}$.

Equation (17) is used to investigate the behavior of systems which are primarily linear but a

nonlinearity is introduced as a perturbation. It also arises in other physical applications. In

(Qu, 1988), three types of symmetry reductions of equation (17) were derived and it was shown tha the equation is unintegrable. The soliton solutions of some special cases of equation (17) were obtained in (Berryman, 1976), (Bona, 1975), ( Liu, 1993), (Makhankov, 1978). by various techniques. 
Table 1: Commutator table of the Lie algebra of (1).

\begin{tabular}{llllll}
\hline$[]$, & $X_{11}$ & $X_{12}$ & $X_{13}$ & $X_{14}$ & $X_{15}$ \\
\hline$X_{11}$ & 0 & 0 & $X_{11}$ & 0 & $X_{14}$ \\
$X_{12}$ & 0 & 0 & $\frac{1}{2} X_{12}$ & 0 & 0 \\
$X_{13}$ & $-X_{11}$ & $-\frac{1}{2} X_{12}$ & 0 & $-\frac{1}{2}\left(\frac{n-3}{n-1}\right) X_{14}$ & $\frac{1}{2}\left(\frac{n+1}{n-1}\right) X_{15}$ \\
$X_{14}$ & 0 & 0 & $\frac{1}{2}\left(\frac{n-3}{n-1}\right) X_{14}$ & 0 & 0 \\
$X_{15}$ & $-X_{14}$ & 0 & $-\frac{1}{2}\left(\frac{n+1}{n-1}\right) X_{15}$ & 0 & 0
\end{tabular}

\section{SYMMETRY REDUCTION EXACT/INVARIANT SOLUTION}

AND

One of the main objectives for calculating the symmetries of differential equations is to use

them to reduce the differential equations which could be solved to obtain exact solutions.

In this section, we apply the symmetries calculated in Section 3 to obtain reduced wave equations and exact solutions where possible. In particular, we use the translation generators in $t$ variable given as $X_{22}=\frac{\partial}{\partial t}$

Case (i) $n=1$

Case (i) $\mathbf{n}=\mathbf{1}$

The reduction using $X_{22}$ reduces (15) to fourth order linear ordinary differential equation in $x$

given as

$w u^{t s s s}-\gamma u^{t s}=0$

whose general solution is

$u(x)=C_{11} e^{-x \sqrt{\frac{P}{x}}}+C_{2} e^{x \sqrt{\frac{P}{x}}}+x C_{9} x \quad C_{1}, C_{2}, C_{9}$ and $C_{4}$ are constants.

Case (ii) $n=2$

With the same generator $X_{22}$, (1) reduces to the following fourth order non linear ordinary

differential equation in $x$ variable

$\omega u^{t s s s}-2 \gamma u^{t s} u^{s}=0$.

The solution of (20) is

$u(x)=C_{1}-$

$6^{\frac{2}{3}}$ Weierstrass Zeta $\left[\frac{\left(\frac{2}{W^{\frac{1}{3}}\left(x+C_{2}\right)}\right.}{\frac{1}{6^{3}}},\left\{\frac{2 \times 6^{\frac{1}{3}} C_{3}}{\frac{1}{3}}, C_{4}\right\}\right]$

Case (iii) $n=3$

Similar approach reduces (17) to

$\omega u^{t r s y}-3 y u^{t s} u^{v 2}=0$.

The general solution of (21) is an elliptic function given as, $u(x)=\frac{2}{a} \sqrt{2}\left(\sqrt{\alpha}\left(\frac{Y}{c_{1}}\right)^{-\frac{1}{4}}\right.$ Elliptic $F\left(N\left(\frac{Y}{c_{1}}\right)^{\frac{1}{4}}, I\right)+x+$ $\left.C_{2}\right)^{\frac{3}{2}}+C_{3 x}$

where $C_{1}, C_{2}$ and $C_{9}$ are constants. constants.

\section{THE HIDDEN SYMMETRIES}

Since we are interested in improving the solutions of the wave equations, it may also be necessary to look into some hidden symmetries of the wave equations.

It has been found that the reduced equation can admit more Lie point symmetries than the

ones of the original equation. These new Lie point symmetries have been termed Type II

hidden symmetries. On the other hand, it is possible that the reduced equation loses a Lie

point symmetry admitted by the original equation. This lost symmetry is called Type I hidden

symmetry (Abraham-Shrauner \& Govinder, 2006), (Abraham-Shrauner \& Govinder, 2008).

Case (i) $n=1$

The Lie point symmetries of the reduced equation (19) are

$H_{21}=\frac{\partial}{\partial x}, H_{22}=\frac{\partial}{\partial u}, H_{2 a}=u \frac{\partial}{\partial u}, H_{24}=x \frac{\partial}{\partial u}, H_{25}=$

$e^{-\sqrt{\frac{P}{x}}} x \frac{\partial}{\partial u}, H_{2 \sigma}=e^{\sqrt{\frac{\sqrt{P}}{x}}} x \frac{\partial}{\partial u}$.

The symmetries of (19) $H_{21}, H_{22,} H_{23}$ and $H_{24}$ are inherited symmetries from $X_{21}, X_{23}$ and $X_{24}$ of (15) respectively. The remaining two symmetries $H_{25}$ and $H_{26}$ are new symmetries obtained from the reduction and are referred to as Type II hidden symmetries.

Case (ii) $n=3$

From the reduced equation (20), we obtain the following Lie point symmetries

$H_{\mathrm{a} 1}=\frac{\partial}{\partial x}, H_{\mathrm{a} 2}=\frac{\partial}{\partial u}, H_{\mathrm{a} a}=u \frac{\partial}{\partial u}-x \frac{\partial}{\partial x}$

The reduced equation (21) inherits all the symmetries $H_{31}, H_{32}$ and $H_{33}$ from $X_{32}, X_{34}$ and $X_{33}$ of (17) respectively. Therefore, there is no Type II hidden symmetry, instead we have Type I hidden symmetries and they are $X_{31}$ and $X_{35}$

\section{DISCUSSION AND CONCLUSION}

In this paper, a nonlinear PDE found in elasto-plastic ow is studied using the group methods.

The Lie point symmetries of the model equations were derived. We found that the analysed model does not admit space dilation type symmetries as a result of the linearity of the equation when $n=1$. Using the Lie point symmetries, we gave some symmetry reductions and obtained the invariant solutions of the equations.

Furthermore, the hidden symmetry approach was applied to generate more new solutions that would not be predicted from the Lie point symmetries of the original wave equations. 
These results can be used to study deformity in elastic and plastic medium.

\section{REFERENCES}

[1] Abraham-Shrauner, B. \& Govinder, K. S. (2006). Provenance of Type II Hidden Symmetries from Nonlinear Partial Differential Equations, Journal of Nonlinear Mathematical Physics. 13(4), 612-622.

[2] Abraham-Shrauner , B. \& Govinder, K. S. (2008). Master Partial Differential Equations for a Type II Hidden Symmetry, Journal of Mathematical Analysis and Applications, 343(1), 525-530.

[3] Ames, W.F. (1972) . Nonlinear Partial Differential Equations in Engineering, Academic Press, New York

[4] Berryman, J. (1976). Stability of solitary waves in shallow water, Physical Fluids, 19, 771-777.

[5] Bona, J. L. (1975). On the Stability Theory of Solitary Waves, Proceedings on Royal Society London A344, 363-374.

[6] Chen, G.W., \& Yang, Z.J. (2000). Existence and Nonexistence of Global Solutions for a Class of Nonlinear Wave Equations. Mathematical Methods of Appllied Science, 23, 615-631.

[7] Ghil, M. and Paldor, N. (1994). A Model Equation for Nonlinear Wavelength Selection and Amplitude Evolution of Frontal Waves, Journal of Nonlinear Science. 4, 471-496.

[8] Kara, A.H., \& Mahomed, F. M. (1997). Action of Lie-Backlund symmetries on conservation laws. in Proceedings of the 10th International Conference on Modern Group Analysis, 7, Norway (Nordfjordeid ):

[9] Kuramoto, Y. \& Tsuzuki, T. (1976). Persistent propagation of concentration waves in dissipative media far from thermal equilibrium, Progressive Theoretical Physics, 55,.356-369.

[10] Liu, Y. (1993). Instability of Solitary Wave for Generalized Boussinesq Equations, Journal of Dynamical Differential Equations, 5(3), 537-558.

[11] Makhankov, V. G. (1978). Dynamics of Classical Solitons (in Non-Integrable Systems). Phys. Rep. 35120-128.

[12] Naz, R., Mahomed, F. M.\& Mason, D. P. (2008). Comparison of Different Approaches to Conservation Laws for some Partial Differential Equations in Fluid Mechanics, Applied.Mathematics and Computation, 205, 212-230.

[13] J. E. Okeke, R. Narain and K. S. Govinder, (2018) New exact solutions of a generalised Boussinesq equation with damping term and a system of variant Boussinesq equations via double reduction theory; Journal of Applied Analysis and Computation Volume 8, Number 2, April 2018, pages 471-485

[14] J. E. Okeke, R. Narain and K. S. Govinder, (2019) A group theoretic analysis of the generalized Gardner equation with arbitrary order nonlinear terms; Journal of Mathematical Analysis and Applications 479 pages $1967-1985$

[15] Olver, P. J. (1993). Applications of Lie Groups to Differential Equations, Springer, New York, NY, USA.

[16] Peire, A., An, L.J. (1995). A Weakly Nonlinear Analysis of Elasto-Plastic-Microstructure Models. SIAM Journal of Appllied Mathematics, 55 136-155.

[17] Qu, C. Z. (1988). Symmetry Reductions and Integrability for the Modified Boussinesq Equation from EPU Problem, Communications in Theoretical Physics (Beijing, China) 29, 153-156.

[18] Sivashinsky, G. I., Nicolaenko, B., \& Zaleski, S. (1977). Nonlinear analysis of Hydrodynamic Instability of Laminar flames, part 1.Derivation of basic equations. Acta Astronony, 4, 1177-1206.

[19] Wu, H., \& Fan, T. (2007). New Explicit Solutions of the Nonlinear Wave Equations with Damping. Journal of Applied Mathematics, 91, 457-465.

[20] Yang, Z.J., Chen, G.W. (2000). Initial Value Problem for a NonlinearWave Equation with Damping Term. Acta Mathematicae Applicae Sinica, 23 (45), in Chinese.

[21] Yan, Z.Y. (2000). Similarity Reductions for a Nonlinear Wave Equation with Damping Term. Acta Physica Sinica, 49, 2113-2117 in Chinese.

[22] Zhang, H.W., \& Chen, G.W. (2003). Potential Well Method for a Class of Nonlinear Wave Equations of Fourth-Order. Acta Mathematicae Science, 23A, 758-768. 